\title{
Melbourne at SemEval 2016 Task 11: Classifying Type-level Word Complexity using Random Forests with Corpus and Word List Features
}

\author{
Julian Brooke Timothy Baldwin \\ Computing and Info Systems \\ The University of Melbourne \\ jabrooke@unimelb.edu.au \\ tb@ldwin. net
}

\author{
Alexandra L. Uitdenbogerd \\ Computer Science and Info Technology \\ RMIT University \\ sandraudrmit.edu.au
}

\begin{abstract}
SemEval 2016 task 11 involved determining whether words in a sentence were complex or simple for a cohort of people with English as a second language. Training data consisted of 200 annotated sentences, representing the combined judgements of 20 human annotators, such that if any annotator of the group labelled a word as complex, then it was considered to be complex. Testing was based on single annotator judgements. Our system used a random forest classifier with a variety of features, the most important of which were term frequency statistics garnered from four large corpora, and style lexicons built on two large corpora. Minor features in the final system include the presence or absence of words in various readability word lists; many other features we tried were not successful. Our ranking amongst submitted systems did not reflect the strength of our system, due to submitting a far from optimal weighting between complex and simple, but we show that when a more appropriate weighting is used, our system ranks amongst the best submitted systems.
\end{abstract}

\section{Introduction}

Most work related to readability measurement (Chall and Dale, 1995) focuses on text-level assessment, but it is clear that being able to determine the difficulty of individual words is important to both that task as well as related ones such as lexical text simplification (Shardlow, 2014). Although some words can be considered conceptually difficult - that is, a level of intellectual sophistication is required to grasp its meaning - for language learners, it is more common for words to be considered difficult (or complex) simply because a reader has had little or no exposure to them. This exposure may depend on many different external factors related to the person's background, some of which may generalize across other similar readers, while others may be entirely idiosyncratic to the reader in question. For example, those who study academic English, or operate in an academic environment, have a different vocabulary exposure to those who specialize in hospitality English. Therefore, there is value in not only trying to predict some prior difficulty of a word, but also trying to generalize across readers in a similar cohort. Task 11 of the 2016 SemEval competition (Complex Word Identification) is aimed at addressing this challenge.

This paper describes our system for the task. We commenced with previous work in word readability scoring (Brooke et al., 2012) and stylistic lexicon creation (Brooke and Hirst, 2013; Brooke and Hirst, 2014). For features, we drew on a diverse set of corpus-based and human-derived metrics, and built a random forest-based classifier. While a mistake related to the proper distribution of complex versus simple words prevented us from scoring amongst the top teams in either of the evaluations metrics used in this task, we show that by appropriate class weighting with the same classifier and features, we can obtain results on either metric that are competitive or better than the best teams.

\section{Background}

The motivation for SemEval 2016 Task 11 was the need to automatically identify complex 
words (Shardlow, 2013) for the task of automated text simplification (Shardlow, 2014). However, the modelling of word complexity and text complexity has a long history, much of it using the term readability, and intended for finding reading material of an appropriate level of difficulty for children and language learners (Chall and Dale, 1995). The measurement of word readability, or lexical complexity, is a fundamental component for a range of techniques and their applications beyond automated text simplification: text readability measurement is used as a basis for automatically recommending reading to language learners (Collins-Thompson and Callan, 2004a); lexical complexity measurement can also allow the automatic glossing of reading material presented electronically (Walmsley, 2011), or the display of text comprising a mixture of two languages (Uitdenbogerd, 2014).

Early work on readability resulted in a large number of measures being developed, typically based on tests given to school-aged native speakers of English (Klare, 1974). The majority of these measures had two recognizable components: grammatical difficulty and word difficulty. The word difficulty component of the measures had the following varieties: inclusion in a list of generally known words, such as the Dale-Chall measure (Chall and Dale, 1995); word length in letters, such as the Automated Readability Index (Senter and Smith, 1967) and Coleman-Liau formulae (Coleman and Liau, 1975); word length in syllables, such as the Flesch and Kincaid formulae (Kincaid et al., 1975); the proportion of words exceeding a word length threshold in characters, such as Lix; and proportion of words exceeding a length threshold in syllables, such as the SMOG formula (Klare, 1974). Criticism of these early readability measures included their inability to capture conceptual difficulty (Gordon, 1980), leading the field to be abandoned to some extent until the current millennium, in which corpus-based techniques, language models, and classifiers became popular, and large-scale corpora became readily available (Collins-Thompson and Callan, 2004b; François, 2009).

For second language learners, it has been observed that higher frequency of exposure increases the chance that a word is known, leading to a typical vocabulary knowledge profile in which the per- centage of known words per 1000 in a ranked list by frequency, monotonically decreases (Meara, 1992). When language learning is optimized based on word frequency, as is often recommended by researchers of language acquisition (Sinclair and Renouf, 1988), the effect may even be exaggerated.

\section{Data}

The training data released for this task included about 200 sentences, in which each word token that was not a proper noun was annotated as either complex or simple. A word was considered complex if any one annotator from a set of 20 annotators marked it as such. We found many sentences being tagged entirely or almost entirely as complex, for no obvious reason; for training, we excluded any sentence where the number of complex words was greater than or equal to the number of tagged words minus 2, leading to 29 sentences being removed. We also excluded from training any appearances of a set of 140 closed-class function words, which we always classified as simple; both test and training data have words from our list that were tagged as complex, but these appear to be mostly errors, and in general we didn't want our classifier focusing on classifying extremely common words.

After applying these two filters, the total number of tokens tagged complex in the training set was 427 , and the total number of tokens tagged simple was 1234 , or roughly a 3 to 1 ratio of simple to complex. By contrast, the test set, which was the result of annotation by individual annotators of about 9000 sentences, had a ratio of simple to complex of almost 18 to 1 (after the common words are removed), which is an extreme difference in class distribution; though we expected to see this effect, when we prepared our system we had no good way of estimating its magnitude. Late in the competition, the organizers released individual annotations which allowed for a more accurate estimate of the expected class distribution, but we became aware of that only after the competition was over, and our work here is based on optimizing using the initial class distribution.

\section{Lists of Features}

We divide our feature lists into three categories: major features, minor features, and unused features. 
Major features are those which we believe are essential to the good performance of the model; minor features were helpful in the version of the classifier we used here, based on 20-fold cross-validation in the training set, but the effect was fairly modest; and unused features were not found to be helpful, but we include them for completeness to give a full sense of everything we tried. There are too many features (and too many combinations) to offer up individual numerical analysis of what worked and what didn't. Our features were selected by optimizing G-score (see Section 6) with a 20-fold cross-validation of the training set.

\subsection{Major Features}

Term frequency statistics We collected term frequency statistics from four large corpora: the British National Corpus ("BNC": Burnard (2000)), the Gigaword corpus (Graff and Cieri, 2003), the International Conference on Web and Social Media (ICWSM) blog corpus (Burton et al., 2009), and Project Gutenberg (read using the GutenTag tool (Brooke et al., 2015)). We consciously chose corpora that had significant variety with respect to their genre, with the intent of allowing the classifier to focus in on particular kinds of words that certain groups might have trouble with. Note that we typically used the count for the specific word type, but where it didn't exist in the corpus we substituted the lemma count, rather than giving a count of zero. All of the corpora were of benefit to the final model.

Six style lexicons For the ICWSM and the Project Gutenberg corpora, we built lexicons for six lexical styles using the co-occurrence information in these corpora. The six styles are: literary, abstract, objective, colloquial, concrete, and subjective; each style for each corpus is an individual feature. We used the seed set for the six styles from Brooke et al. (2013), and the co-occurrence profile ranking approach from Brooke and Hirst (2014). We chose these two corpora because they are extremely large, varied in content, and we have used and evaluated them in other work; highly constrained language like the newswire text in the Gigaword corpus is unlikely to be of much use for building stylistic lexicons in this fashion.

\subsection{Minor Features}

Dale-Chall List The presence or absence of the word in the Dale-Chall list, a list of 3000 common words used in the Dale-Chall readability metric (Chall and Dale, 1995).

Academic Word List The ranking of the word on the 570-word Academic Word List, which divides academic language into 10 frequency categories (Coxhead, 2000).

Beginner List A list of 4636 beginner words, including words from the Dolch list (Dolch, 1936), previously used as a training/test set in earlier lexical readability work (Brooke et al., 2012).

Is Lemma A boolean feature indicating whether a word is its lemma or not. For instance run is a lemma but ran is not.

\subsection{Unused Features}

Document Frequency We tested document frequency as a complement or alternative to term frequency for the various corpora.

Average Sentence Length The average sentence length of the documents the word appears in, for the 4 corpora used for term frequency. It was a useful feature in our earlier work on lexical readability (Brooke et al., 2012), and is an excellent readability feature generally (Uitdenbogerd, 2005).

Word Length The length of the word, in characters, was useful in early iterations but not in the final model.

Average Word Length The average word length in the documents that the word appears in, for the 4 corpora, is another feature from Brooke et al. (2012).

Formality lexicon The formality lexicon built from the ICWSM corpus in Brooke and Hirst (2014). We believe the information in it overlaps considerably with the 6-style lexicon.

Readability rank The readability rank of words as given by the model from Brooke et al. (2012). 
Complexity lexicon Using the words from the training set, we attempted to build a complexity dictionary using the method of Brooke and Hirst (2014). The results were not competitive when the six-style lexicon was included.

Latinate affixes A boolean feature which indicates the presence or absence of a Latinate affix, which can indicate increased formality (Brooke et al., 2010).

Number of Senses The number of senses of the lemma of the word in WordNet (Fellbaum, 1998).

Hyphen fix For hyphenated words, derive all other statistics using the first word in the hyphenation, instead of the whole word.

Bigram style lexicon The features we use do not distinguish between the word type in different contexts, so we cannot distinguish between word senses. We saw examples of this in the data, such as the word tried in the legal sense in was tried for murder. We made an initial attempt at integrating this information by building a bigram style lexicon, using the same method as the regular method, averaging the styles of the two possible contexts, and either replacing the word style or including it as a different feature. However, performance was worse.

Is Cognate While not actually implemented, we considered the possibility of using cognates, which might allow us to discount otherwise complex words which are easy for L2 speakers coming from European language backgrounds because there is a very similar word in other languages (Uitdenbogerd, 2005). However, the results of our early investigation suggested that cognates were not appearing with greater frequency among the simple words than expected, and that therefore the language background of the participants was probably not uniformly European.

\section{Classifier}

Though rarely competitive on large feature sets, a decision-tree-based classifier has several advantages, being considered by some as the only true off-the-shelf classifier (Hastie et al., 2008), in part because it does not require the feature scaling that is typical in linear classification models, and naturally mixes boolean, ordinal and continuous features without having to convert one to the other. If the number of examples and features is low enough, Random Forests - an ensemble classifier that builds multiple decision trees using subsets of the examples and features and then combining the individual votes - is a powerful classifier with all the advantages of the basic decision tree (except interpretability). On the basis of 20-fold crossvalidation over the training set, we found that it was the best classifier among a wide range of options (including all the other ensemble classifiers) that we tested in Sci-kit learn (Pedregosa et al., 2011). We tuned the parameters with an initial feature set, carried out feature selection, and then tuned the parameters again, with very little further effect. The only two parameters that were different to the default were the number of estimators (50); and the maximum depth (3). We also tested with different class weights to improve our performance with regard to G-score, which is discussed in the next section. Having class weights penalizes errors for a particular class more, which effectively forces the classifier to guess that class more often, shifting the class distribution.

\section{Evaluation Metrics}

The evaluation for this task includes 3 basic metrics (precision, recall, and accuracy) and two combined metrics based on them (F-score and G-score). For precision and recall, the positive class is COMPLEX, and this is the basis for calculating F-score. G-score, the primary evaluation metric for this competition, is the harmonic mean of precision and accuracy, putting extra emphasis on recall for the COMPLEX class beyond that which is built into the accuracy score. Relevant to this task, the effect of G-score is opposite to the class imbalance problem mentioned earlier: when training on a set where the positive class is over-represented, the resulting classifier will do better on G-score than F-score because it will tend to overestimate the instances of the positive class, improving recall. 


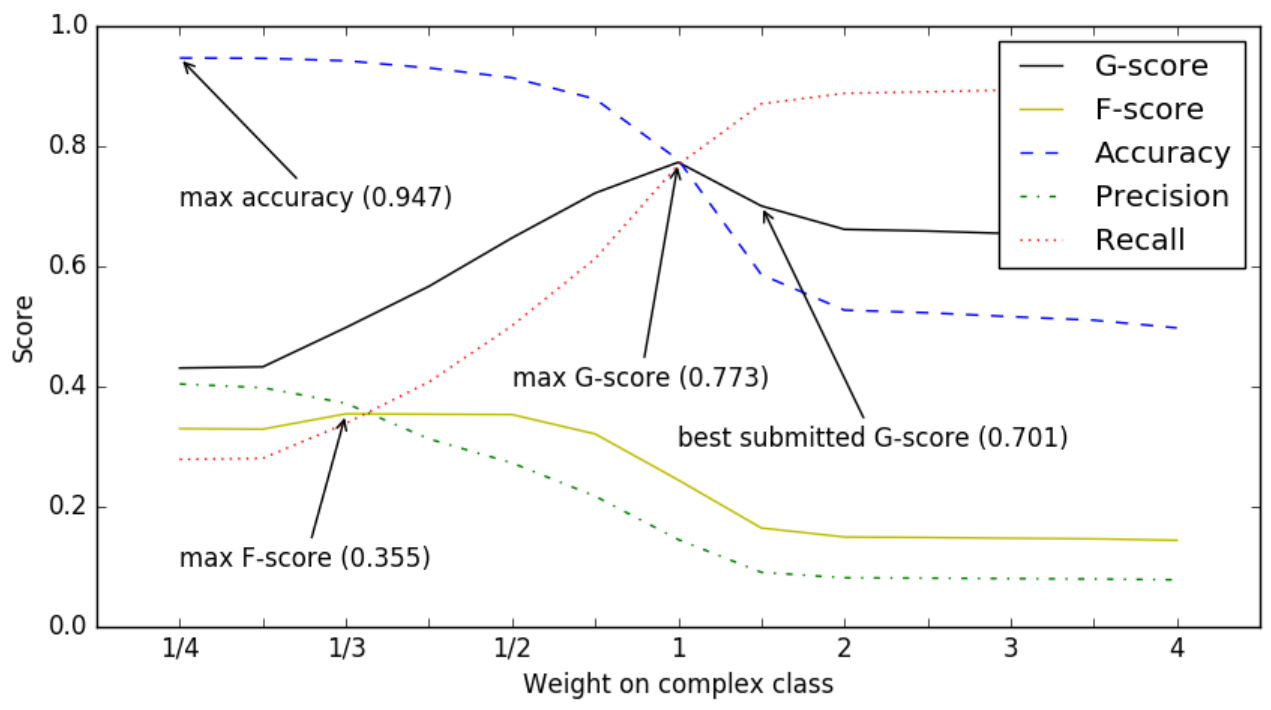

Figure 1: Scores for various metrics for different weightings of the COMPLEX class

\section{Results}

Figure 1 shows the performance of our system for the various metrics across different weightings of the COMPLEX class. With regard to G-score and F-score, our best submitted system is far from optimal, since we overestimated the effect of G-score, and underestimated the influence of the class imbalance between the training and test sets; we incorrectly put too much weight on the COMPLEX class, which resulted in a G-score of 0.701 (12th ranked) for the 1.5 weighting of COMPLEX, and 0.647 (19th ranked) for the 3.0 weighting of COMPLEX. However, across all possible weightings, our best G-score (0.773), which is our system without any weighting at all, is tied with the second best G-score in the competition (the best score was 0.774). If we put more weight on simple words, we reach the maximum F-score of 0.355 when the ratio is (roughly) 3 to 1 in favor of simple words; this F-score is better than any other reported F-score (the best F-score of a submitted system is 0.353 ), though we note that teams might have been more focused on optimizing G-score, since it was the primary metric. Accuracy is maximized simply by minimizing the number of COMPLEX guesses, and in fact guessing only SIMPLE will net an accuracy of 0.953 , which is impossible for our system to beat.

Table 1 shows the results of a small feature ab-

\begin{tabular}{lcc}
\hline Features & G-score & F-score \\
\hline All Features & 0.773 & 0.355 \\
No term frequency & 0.550 & 0.001 \\
No 6-style lexicon & 0.748 & 0.349 \\
No minor features & 0.772 & 0.347 \\
\hline
\end{tabular}

Table 1: Feature ablation

lation study using the best system with regard to each the two combined metrics (no weighting for G-score, 1/3 weighting for F-score); results were erratic for less than optimal values. Term frequency information is clearly the most important source of information for deciding complexity, but we also see improvements due to the stylistic lexicons built using co-occurrence information, and the minor features. The effects are not consistent with respect to degree across the two metrics, likely because different feature sets result in substantially different class distributions, which in turn have very different effects on G-score and F-score.

\section{Discussion}

Our results using the unweighted model put us among the best teams in the competition, though in fact there are 6 other teams with G-scores within 0.01 of each other, and what we know about the effects of weighting should make us cautious about coming to any strong conclusion about which of 
these systems (or other systems, for that matter) are better. From our perspective, it is unfortunate for us that the organizers created a situation where the class distribution in the test set was very unclear. As it happens, the use of G-score almost exactly counters the effect of the class imbalance (in fact, it seems as if G-score may have been selected exactly for this purpose), such that a classifier built on the training data with an eye to F-score will do well with regard to G-score over the testing data (though not the training data), but it didn't seem obvious to us that this would be the case. More generally, we wonder whether some kind of ROC metric might not be more appropriate for this task. In our opinion, the quality of a model of complexity is orthogonal to producing a class distribution which optimizes a particular metric, and collapsing the two just creates confusion and might lead us to overlook otherwise good approaches.

Most of our performance seems to be due to term frequency and word co-occurrence information from a set of four large corpora. Although using multiple corpora was helpful, we actually rather doubt that our model is learning much that is particular to the group of people involved; more likely it is learning a more general model of word difficulty. Choosing the correct proficiency level is primarily a matter of choosing the best class distribution (via weighting); if one has individual annotations of the target population (which we didn't use, but were eventually made available), this is relatively straightforward. What would have been more interesting is if the task had involved multiple groups with very distinct characteristics (for example, two very different L1 language backgrounds, or L1 children versus L2 adults), so that a good model would have had to truly adapt to the specific characteristics of different groups to be successful. It would also be interesting to see if we could build models that can adapt to individuals, predicting words that a reader would or wouldn't know based on a small sample of words tagged by them only. Such a setup might bring us closer to the goals that motivate the task.

\section{References}

J. Brooke and G. Hirst. 2013. Hybrid models for lexical acquisition of correlated styles. In Proceedings of the 6th International Joint Conference on Natural Language Processing (IJCNLP '13), pages 82-90, Nagoya, Japan.

J. Brooke and G. Hirst. 2014. Supervised ranking of co-occurrence profiles for acquisition of continuous lexical attributes. In Proceedings of The 25th International Conference on Computational Linguistics (COLING 2014), pages 2172-2183, Dublin, Ireland.

J. Brooke, T. Wang, and G. Hirst. 2010. Automatic acquisition of lexical formality. In Proceedings of the 23rd International Conference on Computational Linguistics: Poster Volume (COLING '10), pages 90-98, Beijing, China.

J. Brooke, V. Tsang, D. Jacob, F. Shein, and G. Hirst. 2012. Building readability lexicons with unannotated corpora. In Proceedings of the 1st Workshop on Predicting and Improving Text Readability for target reader populations, pages 26-35, Montreal, Canada.

J. Brooke, A. Hammond, and G. Hirst. 2015. GutenTag: An NLP-driven tool for digital humanities research in the Project Gutenberg corpus. In Proceedings of the 4nd Workshop on Computational Literature for Literature (CLFL '15), pages 42-47, Denver, USA.

L. Burnard. 2000. User reference guide for British National Corpus. Technical report, Oxford University.

K. Burton, A. Java, and I. Soboroff. 2009. The ICWSM 2009 Spinn3r Dataset. In Proceedings of the Third Annual Conference on Weblogs and Social Media (ICWSM '09), San Jose, USA.

J. S. Chall and E. Dale. 1995. Readability revisited: the new Dale-Chall readability formula. Brookline Books, Massachusetts, USA.

M. Coleman and T. L. Liau. 1975. A computer readability formula designed for machine scoring. Journal of Applied Psychology, 60(2):283.

K. Collins-Thompson and J. Callan. 2004a. Information retrieval for language tutoring: An overview of the REAP project. In Proc. ACM-SIGIR International Conference on Research and Development in Information Retrieval, Sheffield, UK. Poster.

K. Collins-Thompson and J. Callan. 2004b. A language modeling approach to predicting reading difficulty. In Proceedings of the HLT/NAACL 2004 Conference, pages 193-200, Boston, USA.

A. Coxhead. 2000. A new academic word list. TESOL quarterly, 34(2):213-238.

E. W. Dolch. 1936. A basic sight vocabulary. The Elementary School Journal, 36(6):456-460.

C. Fellbaum, editor. 1998. WordNet: An Electronic Lexical Database. The MIT Press.

T. L. François. 2009. Combining a statistical language model with logistic regression to predict the lexical 
and syntactic difficulty of texts for FFL. In Proceedings of the EACL Student Research Workshop, pages 19-27, Athens, Greece.

R. M. Gordon. 1980. The readability of unreadable text. English Journal, pages 60-61, March.

D. Graff and C. Cieri. 2003. English Gigaword. Linguistic Data Consortium, Philadelphia, PA.

T. Hastie, R. Tibshirani, and J. Friedman. 2008. The Elements of Statistical Learning: Data Mining, Inference and Prediction. Springer, 2 edition.

J. Peter Kincaid, Robert. P. Fishburne Jr., Richard L. Rogers, and Brad. S. Chissom. 1975. Derivation of new readability formulas for Navy enlisted personnel. Research Branch Report 8-75, Millington, TN: Naval Technical Training, U. S. Naval Air Station, Memphis, USA.

G. R. Klare. 1974. Assessing readability. Reading Research Quarterly, X:62-102.

P. Meara. 1992. EFL vocabulary tests. ERIC Clearinghouse.

F. Pedregosa, G. Varoquaux, A. Gramfort, V. Michel, B. Thirion, O. Grisel, M. Blondel, P. Prettenhofer, R. Weiss, V. Dubourg, J. Vanderplas, A. Passos, D. Cournapeau, M. Brucher, M. Perrot, and E. Duchesnay. 2011. Scikit-learn: Machine learning in Python. Journal of Machine Learning Research, 12:28252830.

R. J. Senter and E. A. Smith. 1967. Automated readability index. Technical report, DTIC Document.

M. Shardlow. 2013. A comparison of techniques to automatically identify complex words. In ACL $2013 \mathrm{Stu}$ dent Research Workshop, pages 103-109, Sofia, Bulgaria.

M. Shardlow. 2014. A survey of automated text simplification. International Journal of Advanced Computer Science and Applications, 4(1).

J. M. Sinclair and A. Renouf. 1988. A lexical syllabus for language taching. In R. Carter and M. McCarthy, editors, Vocabulary and language teaching. Longman, London, UK.

A. L. Uitdenbogerd. 2005. Readability of French as a foreign language and its uses. In Australasian Document Computing Symposium, Sydney, Australia.

A. L. Uitdenbogerd. 2014. Tools for supporting language acquisition via extensive reading. In First Workshop on Natural Language Processing Techniques for Educational Applications (NLP-TEA 1), pages 35-41, Nara, Japan.

M. Walmsley. 2011. Automatic glossing for second language reading. In New Zealand Computer Science Research Student Conference 2011, Palmerston North, New Zealand. 